\title{
基于形态分级结构设计的超材料辐射制冷织物
}

\author{
刘辉", 祝世宁 \\ 南京大学物理学院, 固体微结构物理国家重点实验室, 南京 210093 \\ *联系人, E-mail: liuhui@nju.edu.cn
}

\section{Hierarchical-morphology metafabric for scalable passive daytime radiative cooling}

\author{
Hui Liu ${ }^{*} \&$ Shi-Ning Zhu \\ National Laboratory of Solid State Microstructures, School of Physics, Nanjing University, Nanjing 210093, China \\ * Corresponding author, E-mail: liuhui@nju.edu.cn
}

doi: 10.1360/TB-2021-0717

最近几年, 随着全球气候变化, 全球温度升高的趋势越 来越明显, 特别是今年夏天美国高温天气造成多地火灾，阿 联酋甚至出现70多摄氏度的极端高温天气，我国部分地区超 出正常气温的天气也常有发生. 因此, 开发极端炎热天气环 境中的绿色低碳的降温技术, 对于解决气候变化所面临的各 种问题, 具有越来越重要的意义. 个人热管理(personal thermal management)对人体周围微环境的高效调控能力, 可以帮 助人类应对全球变暖背景下的户外极端气候条件. 特别是对 于高热区的户外工作人员来说, 开发一种能够隔热降温的可 穿戴装备, 对于改善工人工作条件具有非常实际的应用价值. 目前, 基于热传导和热对流的设计能够相对有效地实现人体 的热舒适, 但仍不能摆脱耗能、笨重等固有缺陷. 并且这些 相对传统的制冷方案, 其热量转换过程主要利用地表约 $300 \mathrm{~K}$ 的周围环境作为散热源, 进一步限制了其散热效率. 无 源的辐射制冷技术 ${ }^{[1]}$ 则有望解决上述问题: 温度为 $3 \mathrm{~K}$ 的宇宙 空间作为一个“天然冷库”, 为高效的制冷提供了更好的选择. $8 \sim 13 \mu \mathrm{m}$ 中红外波段的大气透明窗口(atmospheric transparent window), 与包括人体在内的地表物体的中红外辐射光谱基 本重叠, 使得利用外太空环境进行制冷成为了可能: 通过选 择性的光谱调控, 面向天空的地表物体可以高效阻挡太阳辐 射, 同时通过大气透明窗口向宇宙辐射热量, 从而通过远程 获取宇宙的寒冷, 实现零能耗的辐射降温. 随着近几年微纳 光学的发展, 研究人员能够利用波长/亚波长尺度的微纳结构 设计, 实现在太阳辐射波段和中红外波段具有选择性光谱特 性的日间辐射制冷系统, 如光子晶体 ${ }^{[2]}$ 、超材料 ${ }^{[3 \sim 5]}$ 和随机介 质 ${ }^{[6,7]}$. 这使得辐射制冷技术有望成为传统耗能热管理技术的 替代及补充方案.
基于辐射制冷技术的人体热管理织物(辐射制冷织物)近 年来也被广泛研究, 在个人热管理领域逐渐受到重视 ${ }^{[8]}$. 但目 前采用不同种类的聚合物所实现的光谱调控范围主要集中 在中红外波段. 这虽能满足热学调控的基本要求，但太阳辐 射波段的吸收使得这类织物在户外暴晒环境难以实现有效 的辐射制冷.

对太阳辐射波段的高效调控, 往往需要在织物内部引人 如空气孔、介质颗粒、聚合物纳米纤维等结构 ${ }^{[9]}$. 但这些方 案在实现高的太阳反射率的辐射制冷织物时仍面临进一步 提高散射效率、降低紫外吸收以及如何提升与工业制备技 术兼容性等挑战 ${ }^{[10]}$. 形态分级的结构设计可以拓宽随机结构 的光谱响应范围, 为实现涵盖太阳辐射波段至中红外波段的 高效宽光谱调控提供了一种可行的解决方案：通过将分级的 随机微纳结构在宏观尺度的空间中进行合理排布，即形态上 的分级，不同级次的结构可以分别响应不同的光谱范围，同 时有效避免不同波段光谱之间的串扰, 优化光谱响应的效率.

华中科技大学陶光明课题组和浙江大学马耀光课题 组 ${ }^{[11]}$ 共同设计制造了一种具有形态分级结构(hierarchicalmorphology structure)的光学超材料织物(metafabric), 以实现 高效的户外个人热管理. 全新结构的超材料织物宏观上体现 为多层织物结构. 其根据织物的多层空间结构、纤维结构以 及纤维内部纳米结构, 在不同的空间、不同的尺度上进行 分级, 形成了一种宏观有序、微观随机的形态学分级体系 (图1(a)). 根据该结构设计，0.3 25 $\mu \mathrm{m}$ 的光谱大体被分解为 3 个波段, 交由metafabric中的不同级次响应. 顶层的聚四氟乙 烯(PTFE)多孔薄膜、底层主体织物中的二氧化钛 $\left(\mathrm{TiO}_{2}\right)$ 纳米 颗粒及微米级的聚乳酸(PLA)聚合物纤维(图1(b), (c)), 分别 

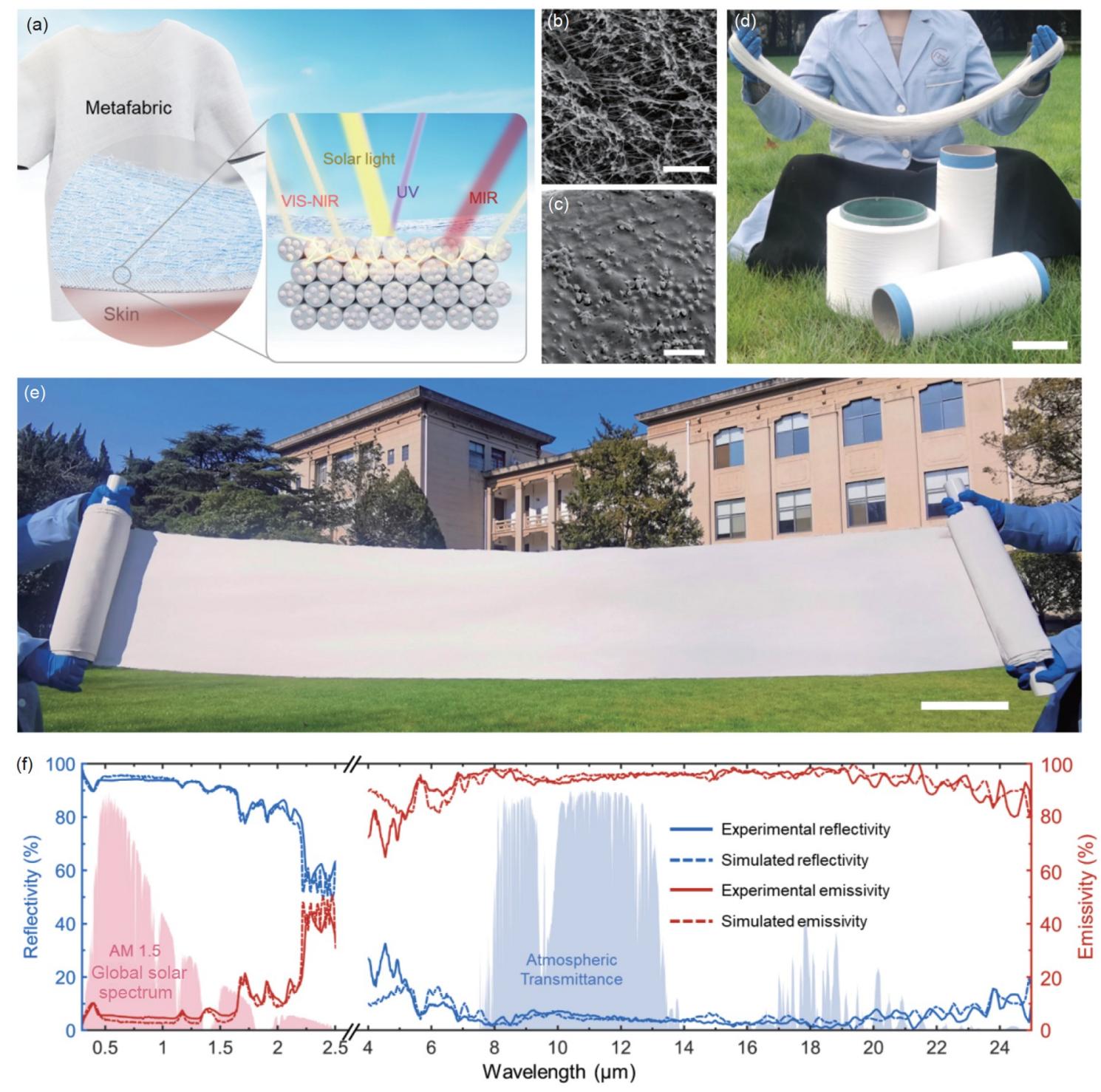

图 1 (网络版彩色)超材料织物的结构和表征 ${ }^{[1]}$. (a) 用于日间辐射冷却的超材料织物示意图; (b) PTFE服装膜的扫描电子显微镜(SEM)图; 比例 尺, $5 \mu \mathrm{m}$. (c) 超材料纤维横截面的SEM图; 比例尺, $5 \mu \mathrm{m}$. (d) 超材料纤维照片; 比例尺, $10 \mathrm{~cm}$. (e) 超材料织物照片; 比例尺, $20 \mathrm{~cm}$. (f) 超材料织物 在0.3 $25 \mu \mathrm{m}$ 的反射率和发射率光谱图

Figure 1 (Color online) Proposed structure and characterization of the metafabric ${ }^{[11]}$. (a) Schematic of a metafabric for daytime radiative cooling. (b) SEM image of the PTFE clothing film. Scale bar, $5 \mu \mathrm{m}$. (c) SEM image of the cross section of the metafiber. Scale bar, $5 \mu \mathrm{m}$. (d) Photograph of the fabricated metafibers. Scale bar, $10 \mathrm{~cm}$. (e) Photograph of the metafabric. Scale bar, $20 \mathrm{~cm}$. (f) Measured reflectivity and emissivity spectra of the metafabric $(0.3-25 \mu \mathrm{m})$

响应紫外、可见-近红外以及中红外波段, 为metafabric提供 了宽谱的太阳反射以及高效的中红外发射.

该研究团队将光学随机超材料与批量纤维制备技术相 结合, 获得了均匀连续的超材料纤维(图1(d)); 进一步利用纺 纱织造和层压技术, 制备得到超材料织物(图1(e)). 项目研究 的metafabric 在太阳光波段 $(0.3 \sim 2.5 \mu \mathrm{m})$ 具有 $92.4 \%$ 的反射 率、在中红外波段(8 13 $\mu \mathrm{m}$ )具有94.5\%的发射率(图1(f)). 基 于可扩展、高通量、全自动制造技术，克服了长期以来将实
验室规模的织物应用于热管理实际场景的挑战. 革新的织物 技术通过分级形态设计, 实现了材料-光学-纺织技术的跨领 域多学科协同创新, 启示并推动传统纺织工业的创新与发展. 超材料织物借助大气透明窗口 $(8 \sim 13 \mu \mathrm{m})$ 增强热量与外部寒 冷空间的热交换, 并对整个太阳辐射波段 $(0.3 \sim 2.5 \mu \mathrm{m})$ 实现高 效的阻挡. 经严格的测试, 在无源输人条件下, 超材料织物可 实现全天低于环境温度 $2 \sim 10^{\circ} \mathrm{C}$ 的良好辐射制冷效果(图2 (a), (b)). 
(a)
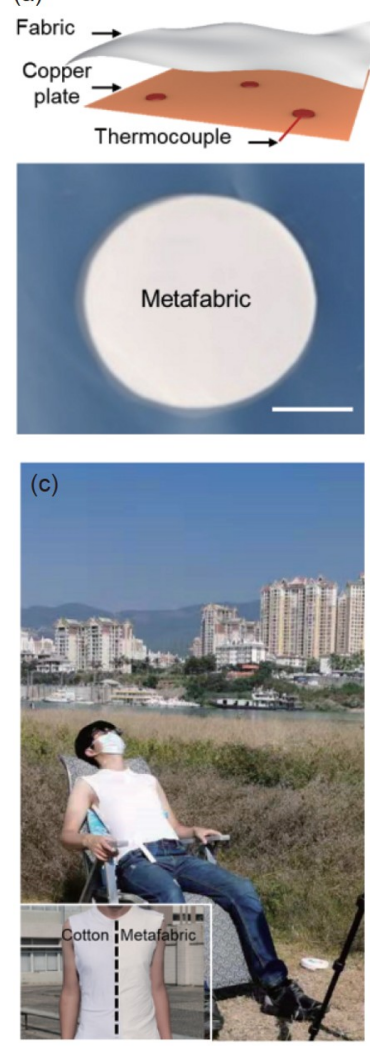
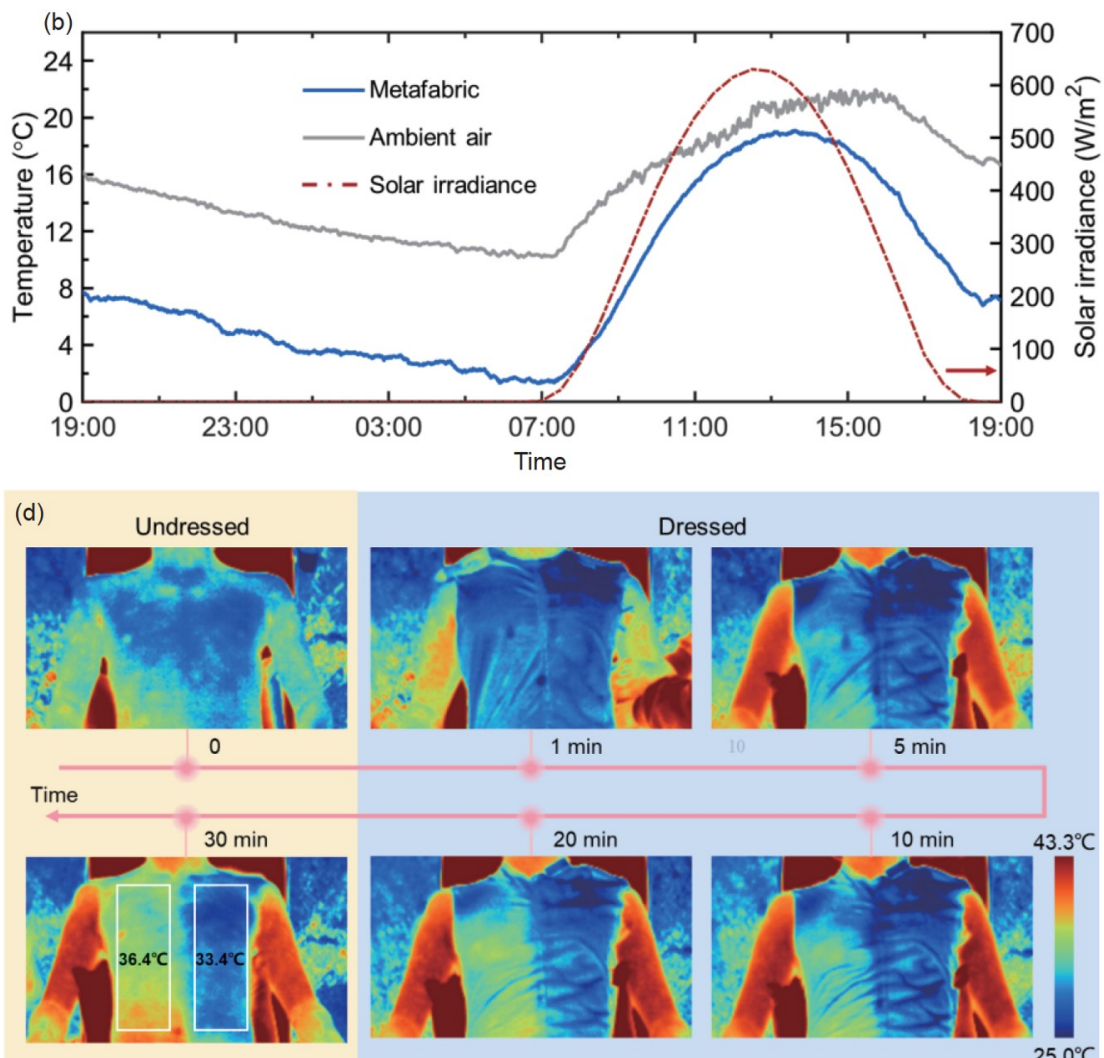

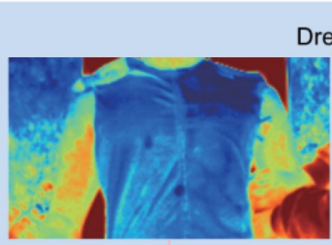

Dressed

图 2 (网络版彩色)超材料织物的降温性能测试. (a) 测试装置示意图和样品照片; (b) 超材料织物 $24 \mathrm{~h}$ 辐射制冷性能测试曲线图(2020年12月 5 6 日, 中国广州, $\left.23^{\circ} 5^{\prime} 32^{\prime \prime} \mathrm{N}, 113^{\circ} 23^{\prime} 45^{\prime \prime} \mathrm{E}\right)$; (c) 超材料织物人体降温对比测试照片; (d) 超材料织物人体降温对比测试红外图(2020年12月 13 日, 中国 西双版纳, $\left.22^{\circ} 10^{\prime} 22^{\prime \prime} \mathrm{N}, 100^{\circ} 51^{\prime} 29^{\prime \prime} \mathrm{E}\right)$

Figure 2 (Color online) Measurement of metafabrics cooling properties. (a) Schematic and photo of the sample structure for the test. Scale bar, 5 cm. (b) A 24-h continuous temperature measurement of the sub-ambient cooling performance test in Guangzhou, China $\left(23^{\circ} 5^{\prime} 32^{\prime \prime} \mathrm{N}, 113^{\circ} 23^{\prime} 45^{\prime \prime} \mathrm{E}\right.$, December 5-6, 2020). (c) Photograph of the metafabric cooling tests on the human body. (d) Infrared images of the volunteer under direct sunlight in Xishuangbanna, China $\left(22^{\circ} 10^{\prime} 22^{\prime \prime} \mathrm{N}, 100^{\circ} 51^{\prime} 29^{\prime \prime} \mathrm{E}\right.$, December 13, 2020)

绿色环保、柔软亲肤、舒适透气的超材料织物不仅具 有良好的日间辐射制冷能力，还具有显著的人体降温效果. 在正午时间段的模拟人体测试中(广州, 2020年11月28日), 超 材料织物所覆盖的模拟皮肤相较于同色商业面料可降温 $5 \sim 7^{\circ} \mathrm{C}$. 在模拟汽车测试中(广州, 12 月 7日), 覆盖超材料织物 的汽车模型内部温度相较于市售车罩的温差超过 $27^{\circ} \mathrm{C}$. 同时, 在人体表面降温测试中, 相较于商用白色棉, 超材料织物最
高降温超过 $4^{\circ} \mathrm{C}$ (广州, 12 月 7 日). 研究团队进一步利用红外相 机记录了人体体表的温度变化(图2(c), 西双版纳, 12月13日). 结果显示, 在长达 $30 \mathrm{~min}$ 的日照后, 志愿者前胸左右两侧体表 呈现出明显温差(图2(d)). 本研究提出的基于形态学分级设计 的超材料织物，为辐射制冷织物的设计提供了一种全新的思 路，为实现极端环境下的户外个人热管理提供了一种低碳环 保的、低成本的、可批量制备的解决方案.

\section{参考文献}

1 Raman A P, Anoma M A, Zhu L, et al. Passive radiative cooling below ambient air temperature under direct sunlight. Nature, 2014, 515: 540-544

2 Chen Z, Zhu L, Raman A, et al. Radiative cooling to deep sub-freezing temperatures through a 24-h day-night cycle. Nat Commun, 2016, 7: 13729

3 Hossain M M, Jia B, Gu M. A metamaterial emitter for highly efficient radiative cooling. Adv Opt Mater, 2015, 3: 1047-1051

4 Zhai Y, Ma Y, David S N, et al. Scalable-manufactured randomized glass-polymer hybrid metamaterial for daytime radiative cooling. Science, 2017, 355: 1062-1066

5 Zhang X, Zhang Z, Wang Q, et al. Controlling thermal emission by parity-symmetric fano resonance of optical absorbers in metasurfaces. ACS Photonics, 2019, 6: 2671-2676 
6 Huang Z, Ruan X. Nanoparticle embedded double-layer coating for daytime radiative cooling. Int J Heat Mass Transfer, 2017, 104: 890-896

7 Mandal J, Fu Y, Overvig A C, et al. Hierarchically porous polymer coatings for highly efficient passive daytime radiative cooling. Science, 2018, 362: 315-319

8 Hsu P C, Song A Y, Catrysse P B, et al. Radiative human body cooling by nanoporous polyethylene textile. Science, 2016, 353: 1019-1023

9 Li D, Liu X, Li W, et al. Scalable and hierarchically designed polymer film as a selective thermal emitter for high-performance all-day radiative cooling. Nat Nanotechnol, 2021, 16: 153-158

10 Weng W, Yang J, Zhang Y, et al. A route toward smart system integration: From fiber design to device construction. Adv Mater, 2020, 32: 1902301

11 Zeng S, Pian S, Su M, et al. Hierarchical-morphology metafabric for scalable passive daytime radiative cooling. Science, 2021, 373 : 692 